\title{
Research on the forecast of tourism reception consumption trend - Taking Shandong Province as an example
}

\author{
Diao Yanhua ${ }^{1, *}$ \\ ${ }^{1}$ School of Economics and Management, Shandong Youth University of Political Science, Shandong Jinan, China
}

\begin{abstract}
Based on the data of 2019 large-scale sample survey of domestic tourists and related economic data, this paper analyses the future per capita domestic tourism expenditure of 16 cities in Shandong Province based on the Markov process. It is found that the unbalanced phenomenon of tourism consumption in 16 cities in the province will exist for a long time, which needs to be paid enough attention. However, in the end, the per capita tourism consumption in most cities will enter the advanced stage from the primary stage and the intermediate stage. Finally, according to the analysis, the paper gives countermeasures and suggestions to stimulate the tourism consumption potential in Shandong Province.
\end{abstract}

\section{Introduction}

Tourism industry as a comprehensive industry, the monitoring and forecasting of it is a late start and relatively difficult research field. From the perspective of type, at present, domestic and foreign scholars' research on tourism index based on big data mainly focuses on two aspects: boom index and monitoring index. Foreign studies on tourism climate index mostly focus on the prediction and analysis of international inbound and outbound tourism market. The tourism demand is predicted by Turner (1997) ${ }^{[1]}$. The research field of domestic scholars has expanded from forecasting the demand of inbound tourism market to the climate index of travel agencies and star hotels (Dai et al., 2007; Tang, 2013) ${ }^{[2-3]}$. However, in general, the research results are still in the stage of academic discussion, and the tourism climate index officially released by the official institutions has not been seen. From the data source and model choice, today's big data era background, tourism source and type is quite rich, such as network search data, payment transaction data, express service data, social media, online news, scan data and satellite remote sensing data, and so on, but the current is applied to tourism index prediction research at home and abroad are only focused on Internet search data, model also focuses on traditional econometric model. For example, Li et al. (2017) use Google, Baidu and other network search data combined with traditional econometric model to forecast the tourist flow [4]. Ren et al. (2014) and Wei et al. (2018) use big data to construct tourism indexes and make predictions [5-6]. Fan (2010), He et al. (2015) and Tang (2015) use Markov model for prediction [7-9]. The application of the existing research on tourism big data is to introduce the search data into the traditional time series model or econometric model to compare its prediction effect with the benchmark model that does not include search data.
The existing research on the tourism consumption market mainly carries out the monitoring and prediction on the macro and micro levels from the comprehensive perspective. There are few researches on the analysis and prediction of the tourism reception consumption, and there is a lack of systematic and detailed research. Therefore, this study takes Shandong Province as the research object. Based on the relevant data of Shandong Provincial Department of Culture and Tourism, the paper predicts the future change trend of tourism in Shandong Province, and provides the basis and reference for relevant departments to formulate incentive policies of tourism consumption scientifically and effectively stimulate the potential of tourism consumption.

\section{Methods and data sources}

\subsection{Markov process prediction}

Markov process, named after Markov, is a special method to describe the development process of things, which is mainly used to predict the development state of things. As is known to all, the state of development of things is always changing with the passage of time. For the development of some things, it is necessary to comprehensively examine their past and present state in order to predict their future. But the development of some things, by knowing the present state, can predict the future state, without knowing the past state of things. The basic method of Markov prediction is to use the transition probability matrix between states to predict the state of the occurrence of the event and its development trend, its basic requirement is that the state transition probability matrix must have a certain stability. Therefore, the accuracy and veracity of prediction can be guaranteed only when enough statistical data are mastered.

\footnotetext{
*Corresponding author: 99581735@qq.com
} 


\subsubsection{Probability of state transition}

In the process of development and change of events, the possibility of starting from a certain state and moving to other states at the next moment is called state transition probability. Assuming the probability of $E_{i}$ state transition to state is $E_{j}$, then. $\mathrm{P}_{\mathrm{ij}}=\mathrm{P}\left(\mathrm{E}_{\mathrm{j}} \mid \mathrm{E}_{\mathrm{i}}\right)$

\subsubsection{State transition probability matrix}

There are n possibilities for the occurrence of the event, i.e., the possible state of the event is. $E_{1}, E_{2}, \cdots, E_{n}$, $P_{i j}$ is the probability of the event being transferred from state $E_{i}$ to state $E_{j}$, then the probability matrix of state transition is:

$$
\mathrm{P}=\left[\begin{array}{llll}
\mathrm{P}_{11} & \mathrm{P}_{12} & \cdots & \mathrm{P}_{1 \mathrm{n}} \\
\mathrm{P}_{21} & \mathrm{P}_{22} & \cdots & \mathrm{P}_{2 \mathrm{n}} \\
\cdots & \cdots & \cdots & \cdots \\
\mathrm{P}_{\mathrm{n} 1} & \mathrm{P}_{\mathrm{n} 2} & \cdots & \mathrm{P}_{\mathrm{nn}}
\end{array}\right]
$$

If one of the predicted events is currently in a state $E_{i}$, then at the next moment, it may shift from a state $E_{i}$ to any of these states $E_{1}, E_{2}, \cdots, E_{n}$. Therefore, the following conditions are satisfied about $P_{i j}$.

$$
\left\{\begin{array}{l}
0 \leq P_{i j} \leq 1(i, j=1,2 \cdots, n) \\
\sum_{i=1}^{n} P_{i j}=1(i, j=1,2 \cdots, n)
\end{array}\right.
$$

If $\mathrm{P}$ is a probability matrix, and there is an integer $\mathrm{m}$ $(>0)$, such that all elements in the probability matrix $P_{m}$ are non-zero, then $\mathrm{P}$ is called the standard probability matrix. It can be proved that if $\mathrm{P}$ is the standard probability matrix, then there exist non-zero vectors $a=$ $\left[x_{1}, x_{2}, \cdots, x_{n}\right]$, where, $x_{i}$ satisfies the conditions $0 \leq$ $x_{i} \leq 1$, and $\sum_{i=1}^{n} x_{i}=1$, that

$$
l_{j}(k)=\left(l_{1}(k), l_{2}(k) \cdots, l_{n}(k)\right)
$$

Assuming row vectors $l(k)=\left(l_{1}(k), l_{2}(k) \cdots, l_{n}(k)\right)$, the recursive formula for calculating state probability successive times can be obtained from Equation 3.

$$
\left\{\begin{aligned}
l(1) & =l(0) P \\
l(2) & =l(1) P=l(0) P^{2} \\
& \cdots \cdots \cdots \\
l(k) & =l(k-1) P=\cdots \cdots=l(0) P^{k}
\end{aligned}\right.
$$

Among them: $l(0)=\left(l_{1}(0), l_{2}(0) \cdots, l_{n}(0)\right)$

\subsection{Data Sources}

In this paper, the data of tourism consumption forecast mainly comes from Shandong Province tourism data overview.

\section{The consumption trend forecast research of Shandong province}

\subsection{Division of states}

There are 16 cities and prefectures in Shandong Province. This paper uses the data of per capita tourism expenditure in various regions of Shandong Province from 2010 to
2019 to establish the Markov prediction model of per capita tourism expenditure in various regions of Shandong Province. According to the development of tourism in various regions of Shandong Province and combined with relevant literature, this paper divides the per capita domestic tourism expenditure in various regions of Shandong Province into four states, which are as follows:

E1- primary stage (per capita travel spending less than 900 yuan);

E2- intermediate stage (between 900 yuan and 1200 yuan per capita);

E3- middle and advanced stage (per capita tourism spending between 120 and 150 billion yuan);

E4- Advanced stage (over 1,500 yuan per person).

According to the above classification criteria, the cities in Shandong Province were divided into different regions, and the statistical results after division were shown in Table 1.

Table1. Statistics on the types of per capita domestic tourism consumption in different cities of Shandong Province from 2010 to 2019

\begin{tabular}{|c|c|c|c|c|}
\hline Year/State & E1 & E2 & E3 & E4 \\
\hline 2010 & 12 & 3 & 1 & 0 \\
\hline 2011 & 12 & 3 & 1 & 0 \\
\hline 2012 & 12 & 3 & 1 & 0 \\
\hline 2013 & 9 & 6 & 1 & 0 \\
\hline 2014 & 8 & 7 & 1 & 0 \\
\hline 2015 & 7 & 8 & 0 & 1 \\
\hline 2016 & 6 & 8 & 1 & 1 \\
\hline 2017 & 6 & 6 & 3 & 1 \\
\hline 2018 & 5 & 7 & 3 & 1 \\
\hline 2019 & 3 & 9 & 3 & 1 \\
\hline
\end{tabular}

Data source: collated in this study.

\subsection{Establish the state transition probability matrix}

According to the above changes in the number of states, the number of state transitions in each year can be obtained, so that the probability matrix of state transitions in each year can be calculated. The state transition matrices of 2010 -- 2011, 2011 -- 2012, 2012 -- 2013, 2013 -- 2014, 2014 -- 2015, 2015 -- 2016, 2016 -- 2017, 2017 -- 2018 and 2018 -- 2019 are as follows:

$$
\begin{aligned}
P_{1} & =\left[\begin{array}{llll}
1 & 0 & 0 & 0 \\
0 & 1 & 0 & 0 \\
0 & 0 & 1 & 0 \\
0 & 0 & 0 & 1
\end{array}\right] \\
P_{3} & =\left[\begin{array}{llll}
\frac{8}{9} & \frac{1}{9} & 0 & 0 \\
0 & 1 & 0 & 0 \\
0 & 0 & 1 & 0 \\
0 & 0 & 0 & 1
\end{array}\right] \quad P_{2}=\left[\begin{array}{llll}
1 & 0 & 0 & 0 \\
0 & 1 & 0 & 0 \\
0 & 0 & 1 & 0 \\
0 & 0 & 0 & 1
\end{array}\right]
\end{aligned}
$$




$$
\begin{aligned}
& P_{4}=\left[\begin{array}{cccc}
\frac{7}{8} & \frac{1}{8} & 0 & 0 \\
0 & 1 & 0 & 0 \\
0 & 0 & 0 & 1 \\
0 & 0 & 0 & 1
\end{array}\right] \quad P_{5}=\left[\begin{array}{cccc}
\frac{6}{7} & \frac{1}{7} & 0 & 0 \\
0 & \frac{7}{8} & \frac{1}{8} & 0 \\
0 & 0 & 1 & 0 \\
0 & 0 & 0 & 1
\end{array}\right] \\
& P_{6}=\left[\begin{array}{cccc}
1 & 0 & 0 & 0 \\
0 & \frac{3}{4} & \frac{1}{4} & 0 \\
0 & 0 & 1 & 0 \\
0 & 0 & 0 & 1
\end{array}\right] \\
& P_{7}=\left[\begin{array}{cccc}
\frac{6}{7} & \frac{1}{7} & 0 & 0 \\
0 & \frac{7}{8} & \frac{1}{8} & 0 \\
0 & 0 & 1 & 0 \\
0 & 0 & 0 & 1
\end{array}\right] \quad P_{8}=\left[\begin{array}{cccc}
\frac{5}{6} & \frac{1}{6} & 0 & 0 \\
0 & 1 & 0 & 0 \\
0 & 0 & 1 & 0 \\
0 & 0 & 0 & 1
\end{array}\right] \\
& P_{9}=\left[\begin{array}{llll}
\frac{3}{5} & \frac{2}{5} & 0 & 0 \\
0 & 1 & 0 & 0 \\
0 & 0 & 1 & 0 \\
0 & 0 & 0 & 1
\end{array}\right]
\end{aligned}
$$

In order to reduce random errors and increase the reliability and accuracy of calculation, the state transition probability matrix of each year can be averaged to obtain the following transition matrix:

$$
P=\left[\begin{array}{cccc}
0.8791 & 0.1209 & 0.0000 & 0.0000 \\
0.0000 & 0.9444 & 0.0555 & 0.0000 \\
0.0000 & 0.0000 & 0.8888 & 0.1111 \\
0.0000 & 0.0000 & 0.0000 & 1.0000
\end{array}\right]
$$

\subsection{Prediction model calculation}

Given the state transition probability matrix $\mathrm{P}$, as long as the initial state of the system is solved, the possible state of the system at any moment can be inferred by using the recursive formula. Let $X^{K}=\left(X_{1}^{K}, X_{2}^{K}, X_{3}^{K}, X_{4}^{K}\right)$ as the state number of per capita tourism expenditure in all cities of Shandong Province in the year K, and

$$
X^{0}=\left(X_{1}^{0}, X_{2}^{0}, X_{3}^{0}, X_{4}^{0}\right)=\left(\frac{20}{4}, \frac{30}{4}, \frac{10}{4}, \frac{4}{4}\right)
$$

as the initial state probability (calculated by the average of the four states from 2016 to 2019). By using the formula $X^{K}=X^{0} P^{K}$, we can get:

Number of per capita tourism expenditure in various cities of Shandong Province in 2020

$$
X^{1}=X^{0} P^{1}=(4.3956,7.6875,2.6383,1.2778)
$$

After 10 years, that is, in 2029, the number of tourism expenditure per capita in different cities of Shandong Province

$$
X^{10}=X^{0} P^{10}=(1.3780,6.9053,3.3699,4.3359)
$$

Twenty years later, that is, 2039, the number of tourism expenditure per capita in different cities of Shandong Province

$$
X^{20}=X^{0} P^{20}=(0.3340,0.4221,2.9177,13.3056)
$$

\subsection{Result analysis and Conclusion}

According to the Markov prediction model, 1.2778 regions in Shandong Province would be in the advanced stage in 2020. By referring to relevant information of different cities, it can be seen that in 2019, the per capita tourism cost of Jinan and Yantai was close to 1300 yuan. Yantai has always been a hot tourist city in Shandong because of its superior geographical location, convenient transportation and beautiful mountain and sea scenery. As the capital city of Shandong Province, Jinan is blessed with mountains, springs, lakes and rivers to develop its tourism industry. In recent years, it has been attracting tourists from all over the world through its own influence and unique charm, and its tourism industry has been developing rapidly. After excluding the epidemic factors, the probability of the per capita tourism spending approaching or exceeding 1,500 yuan in 2020 is very high. Therefore, the conclusion drawn is basically consistent with Markov's prediction. According to the Markov prediction model, after 10 years, 1.3780 regions in Shandong Province will be in the primary stage, 3.3699 regions will be in the middle and advanced stage, and 4.3359 regions will be in the advanced stage. As is expected, 20 years later, there will still be 0.3340 areas in the primary stage in Shandong Province. According to the current per capita consumption growth rate of Dezhou, after 20 years of development, it may still be in the primary stage, while Qingdao has entered the developed stage in 2015, which indicates that tourism consumption in Shandong Province presents serious regional imbalance, which needs to be paid enough attention.

Although there are some differences in the tourism consumption of different regions in Shandong Province, according to Markov's ultimate state prediction, the tourism consumption of different cities in Shandong Province will eventually enter the advanced stage. Tourism consumption changes from primary stage, intermediate stage and intermediate stage to advanced stage, which is consistent with the objective law that there is only one final state -- advanced state when calculating the limit of state transfer matrix.

\section{Countermeasures and suggestions}

\subsection{Strengthen facilities construction and improve public tourism services}

First, we will improve the construction of tourist attractions. We will integrate modern technology and cultural creativity to encourage "old trees to sprout new branches". To enhance the brand levels of tourist cities, scenic spots, resort areas, hotels, tourist shopping and tourism services, and build a strong province of tourism brands in China. Second, we will strengthen tourism infrastructure. The government should optimize the route structure, and support Qingdao and Jinan to open more encrypted intercontinental routes, and Yantai, Weihai, Linyi and Rizhao to open more encrypted mainland routes. We will support Jinan, Qingdao, Yantai, Weihai and other international airports in enhancing their gateway functions to Japan and Korea, actively cultivate international airlines to Europe, the United States, Australia, Russia and other countries, moderately encrypting flights to Southeast Asia, and give key support in airline marketing and other aspects. We will promote the development of high-speed railway tourism economic circles, accelerate the construction of trans-regional and key railway economic belts, optimize the arrangement of 
train schedules at tourist destinations according to actual needs, and comprehensively improve the capacity of railway tourism passenger transport. We will actively implement the construction of tourist highways and the project of "highway connecting lines into scenic spots", promote the development of tourist highways, and create the most beautiful highway service areas. Along the highways, trees should be trees, irrigation should be irrigation, and flowers should be flowers. We suggest that the relevant departments rationally arrange tourist service facilities such as service areas, moorings, viewing platforms, post stations and self-drive recreational vehicle camps along national and provincial highways and highways leading to scenic spots.

\subsection{Enrich cultural and tourism formats and promote in-depth integration of cultural and tourism}

First, we will enrich new products and new forms of business in cultural tourism. We will fully implement the "tourism plus" strategy, promote the integration and penetration of tourism with industries such as industry, agriculture, ecology, culture, education, sports and medical care, accelerate innovation in tourism services and products, and extend and upgrade the tourism industry chain and value chain. Second, further strengthen the integration of culture and tourism development of the infrastructure. Third, we will cultivate innovative talents who devote themselves to the integrated development of culture and tourism. In promoting cultural and tourism integration to strengthen development of talent, should especially pay attention to the development of the local talent, on the one hand, take the way of "please come in and go out" to foster cultural tourism professionals, the introduction of foreign or domestic developed regions cultural tourism creative, research and development, management in areas such as high-end talent or invite relevant experts for technical guidance, encourage and organize local cultural tourism practitioners to cultural tourism in developed countries and regions to learn and study. On the other hand, encourage institutions of higher learning according to the needs of the development of cultural and tourism integration set up the disciplines and specialties, cultivate cultural and tourism integration development needs of all kinds of talents, encourage cultural enterprises, tourism enterprises on staff training and re-education, dynamic update employees' knowledge and skills, establish long-term effective mechanism of university-enterprise cooperation, actively carry out cooperation between colleges and universities according to the needs of the enterprises in urgent need of talent cultivation.

\subsection{Excavate local culture and take the characteristic and creative road}

First, to inject culture into rural tourist attractions. Strengthen the forward-looking and innovative tourism projects to meet the increasingly diverse needs of tourists. Second, enhance the diversification of tourism products.
According to the different tourism market, set different tourism products, to meet the needs of different visitors. Third, to strengthen the characteristics, creativity. In terms of characteristics, rural tourism should adhere to its rurality, local nature and uniqueness, so as to distinguish it from urban leisure projects, avoid homogenized competition and form a diversified rural tourism destination. In terms of creativity, we should build a team of cultural and creative talents combining professional talents with local talents. Discover the local culture, take the local local soil as the root to excavate, and graft other cultures appropriately; Create a platform for rural cultural creativity to promote communication, improvement and cooperation.

\subsection{Aggregate innovative ideas and expand product marketing channels}

First, we will build iconic tourism shopping brands, expand both online and offline markets, support the entry of high-quality and featured tourist goods into scenic spots, featured commodity blocks, expressway service areas and other tourist areas, and encourage cooperation with e-commerce companies such as JD and Tmall. Second, we will develop innovative tourism products. We will accelerate the development of new experiential cultural tourism products such as animation, online games, online audio-visual products, and virtual reality. Cultivate traditional craft products with regional characteristics and brand value, develop traditional festival cultural supplies and folk art, folk performance projects.

\section{References}

1. Turner L. (1997). The use of composite national indicators for tourism forecasting. Tourism Economics, 3(4):309-317.

2. Dai B., Yan X, Huang X. (2007) Study on Indexation of China's Travel Service Industry's Business Cycle. Tourism Tribune, 22(9):35-40.

3. Tang CC. (2013). Evaluation and promotion model of tourism climate index in Chinese heritage sites. Resources Science, 35(12):344-351.

4. Li X, Pan B, Law R (2017). Forecasting tourism demand with composite search index. Tourism Management, 59(4):57-66.

5. Ren L, Cui DJ. (2014). Prediction of Domestic Tourist Flow Based on Internet Search Data -- A Case Study of Beijing's Domestic Tourist Flow. Exploration of Economic Problems, 4:67-73.

6. Wei JR, Cui HM (2018). Research on regional tourism index and its micro-dynamics based on Internet search data: A case study of Xi 'an, System science and mathematics, 38 (02): 177-194.

7. Fan GJ. (2010) Tourism Destination Number Prediction Based on Markov Model. Statistics and Decision, 21:98-100.

8. He J, Zhang YL (2015) Tourism revenue prediction 
of Henan Province based on optimized grey-Markov chain. Henan Science and Technology, 4:686-690.

9. Tang R. (2015) Analysis on the tourism consumption structure of domestic tourists to Shanghai based on Markov prediction. Tourism Forum, 1:38-45. 